\title{
Adenocarcinoma de pulmón simulando una enfermedad pulmonar intersticial en un varón de 30 años
}

\author{
S. CASALLO BLANCO, F. MARCOS SÁNCHEZ, L. DE MATÍAS SALCES, \\ A. VIANA ALONSO, J. CELDRÁN GIL, E. NÚÑEZ CUERDA \\ Servicios de Medicina Interna y de Neumología. Hospital Nuestra Señora del Prado. \\ Talavera de la Reina, Toledo
}

LUNG ADENOCARCINOMA FEIGNING AND INTERSTITIAL LUNG DISEASE OF A 30-YEAR-OLD MAN

\begin{abstract}
RESUMEN
Presentamos el caso de un varón de 30 años de edad, con antecedentes de asma bronquial, que presentó un cuadro de tos, disnea, fiebre y dolor torácico.

En la radiografía de tórax se observó cardiomegalia y un patrón intersticial bilateral. En el ecocardiograma, se apreció un derrame pericárdico severo, que obligó a la realización de una pericardiocentésis. En una TAC torácica se observaron adenopatías mediastínicas y un patrón intersticial retículo nodular bilateral. No se pudo completar la broncoscopia por mala tolerancia. Finalmente una biopsia pulmonar permitió el diagnóstico de adenocarcinoma de pulmón.

Posteriormente se comentan algunos aspectos del cáncer de pulmón en pacientes jóvenes (menores de 40 años), destacando datos sobre su incidencia, anatomía patológica, clínica y pronóstico.

Referimos algunos aspectos de la enfermedad intersticial pulmonar y su diagnóstico diferencial con el carcinoma de pulmón con linfangitis carcinomatosa.
\end{abstract}

PALABRAS CLAVE: Adenocarcinoma de pulmón. Enfermedad pulmonar intersticial. Linfangitis carcinomatosa.

\begin{abstract}
A case of a 30-year-old man is presented. He had a past medical history of asthma and presented with cough, shortness of breath, fever and chest pain.

The chest X-ray showed cardiomegaly and a interstitial pattern. The echocardiogram revealed a severe pericardial effusion that required performing a pericardiocentesis. A CT scan showed mediastinal adenopathies and reticulonodular intertitial pattern. A bronchoscopy could not be completed because of non-tolerance.

Finally a lung biopsy allowed us to reach a final diagnosis of lung adenocarcinoma.

Following, some aspects of lung cancer in young people are commented, especially its incidence, histology, symptoms and a prognosis.

A few aspects of the interstitial lung disease are also addressed and the differential diagnosis with lymphangitic carcinomatosis.
\end{abstract}

KEW WORDS: Lung adenocarcinoma. Interstitial lung disease. Lymphangitic carcinomatosis.

Casallo Blanco S, Marcos Sánchez F, Matías Salces L, Viana Alonso A, Celdrán Gil J, Núñez Cuerda E. Adenocarcinoma del pulmón simulando una enfermedad pulmonar intersticial en un varón de 30 años. An Med Interna (Madrid) 2007; 24: $289-291$.

\section{INTRODUCCIÓN}

El cáncer de pulmón es desde hace muchos años la primera causa de muerte por cáncer en los países occidentales. Constituye uno de los problemas de salud de mayor envergadura, por su elevada mortalidad y también a que su diagnóstico se realiza habitualmente en estadios avanzados.

Más de la mitad de los casos se presentan como enfermedad metastásica, un tercio como enfermedad localmente avanzada y sólo un $25 \%$ como enfermedad operable.

En España la incidencia del cáncer de pulmón continua en ascenso en ambos sexos, si bien las tasas son inferiores a las norteamericanas.

La edad media de los pacientes con cáncer de pulmón es alrededor de los 60 años, si bien el rango de edades al diagnóstico es amplio (1).
Presentamos el caso de un varón de 30 años con un adenocarcinoma de pulmón cuyo diagnóstico inicial basándonos en la clínica y en la imagen radiológica, fue de una enfermedad pulmonar intersticial difusa (EPID).

\section{CASO APORTADO}

Varón de 30 años de edad, que un mes antes de su ingreso presentó un cuadro de tos, cefalea y disnea de esfuerzo progresiva. Dos semanas más tarde el paciente presentó expectoración mucopurulenta y fiebre de $38^{\circ} \mathrm{C}$. Acudió a su médico quién le indicó tratamiento con amoxicilina y posteriormente moxifloxacino y bromuro de tiotropio. Consultó a urgencias al presentar disnea de reposo, intenso dolor retroesternal y palpitaciones.

No era fumador y tenía antecedentes de asma bronquial, que trataba con salbutamol inhalado. 
A la exploración física presentaba T.A: 110/70 mmHg, 37,5 C, estaba consciente, taquipneico, sudoroso, no presentaba ingurgitación yugular. En la auscultación pulmonar se objetivaban crepitantes bilaterales difusos y en la auscultación cardiaca tonos rítmicos, rápidos sin soplos ni extratonos. El abdomen era blando depresible y no presentaba organomegalias. En las extremidades no había edemas y los pulsos periféricos estaban conservados.

En cuanto a las pruebas de laboratorio mostraban 18.500 leucocitos con desviación izquierda, hemoglobina de 16,3 gramos/dl, 474.000 plaquetas, actividad de protrombina del 73\%. Dímero D $920 \mathrm{mg} / \mathrm{dl}$. Gasometría arterial basal: pH 7,53, pO2 $47 \mathrm{mmHg}, \mathrm{pCO}_{2} 23 \mathrm{mmHg}$, $\mathrm{CO} 3 \mathrm{H} 19,2 \mathrm{mmol} / \mathrm{l}$. Bioquímica normal incluyendo troponina y CPK.

En la radiografía de tórax se observaba cardiomegalia severa y un patrón intersticial bilateral. Ante estos hallazgos se realizó un ecocardiograma transtorácico en la que se apreció un derrame pericárdico severo, sin datos de taponamiento y un ventrículo izquierdo de dimensiones normales.

Ingreso en la unidad de vigilancia intensiva, iniciándose tratamiento con furosemida, levofloxacino, ácido acetilsalicílico y ventilación mecánica no invasiva.

En un nuevo ecocardiograma se observaron datos de taponamiento, por lo que se efectúo una pericardiocentésis evacuadora, obteniendo un litro de ún líquido serohemático, con las siguientes características bioquímicas: LDH 2353 U/l, proteínas 5,3 gr, 9855 células con un $98 \%$ de polimorfonucleares.

A los siete días fue trasladado al Servicio de Medicina Interna donde se inició el estudio para determinar la etiología de derrame pericárdico y el patrón intersticial, realizándose las siguientes pruebas: anticuerpos antinucleares, factor reumatoide, Mantoux, negativos. El perfil tiroideo fue normal.

El cultivo del líquido pericárdico fue negativo, así como tres hemocultivos.

La anatomía patológica del líquido pericárdico fue informada de inflamación aguda intensa y grupos de células mesoteliales con atípias de aspecto reactivo, siendo negativo para células malignas.

El antígeno carcinoembrionario (CEA) se encontró elevado $21,53 \mathrm{ng} / \mathrm{ml}$. Además se realizaron serologías al virus de la inmunodeficiencia humana, Mycoplasma pneumoniae, citomegalovirus, Epstein Barr y Chlamydia pneumonie, que fueron negativos salvo la $\operatorname{IgM}$ a citomegalovirus que fue positiva.

Una TAC torácico mostró dos adenopatías mediastínicas de tamaño patológico de localización pretraqueal de aproximadamente 19 x $15 \mathrm{~mm}$ y 18 x $17 \mathrm{~mm}$ respectivamente, y un derrame pleural bilateral algo mayor en el lado derecho (Fig. 1). Con ventana pulmonar se observaron alteraciones bilaterales y difusas del parénquima pulmonar con un patrón intersticial retículo nodular septal y centro lobulillar con áreas de condensación alveolar parcheada de distribución predominante en segmento apicales de lóbulos inferiores y ambos lóbulos superiores (Fig. 2).

Se realizó una broncoscopia, observando vías aéreas superiores, cuerdas vocales y traquea sin alteraciones, existía un ensanchamiento en la zona subcarinal. El árbol bronquial no presentaba alteraciones. Se realizó lavado bronquioalveolar en el segmento apical del culmen, teniendo que suspenderse la exploración por mala tolerancia no pudiéndose realizar punción de las adenopatías ni biopsia transbronquial.

La anatomía patológica del lavado informó de la existencia de abundantes macrófagos y grupos epiteliales con atipias de tipo reactivo, siendo compatible con hiperplasia de neumocitos tipo II, secundario a daño pulmonar agudo.

Se pensó que podría presentar una enfermedad pulmonar intersticial difusa(EPID) debido a las características anatomopatológicas, clínica y radiológicas. La primera posibilidad era una neumonía eosinofílica por la presencia de eosinofilia en sangre periférica, iniciándose tratamiento con prednisona $1 \mathrm{mg} / \mathrm{kg}$ de peso siendo la evolución favorable, procediéndose al alta a los 25 días de ingreso, sin fiebre ni disnea.

Siete días más tarde acudió nuevamente por un cuadro de tos con expectoración mucosa y posteriormente hemoptoica. La radiografía

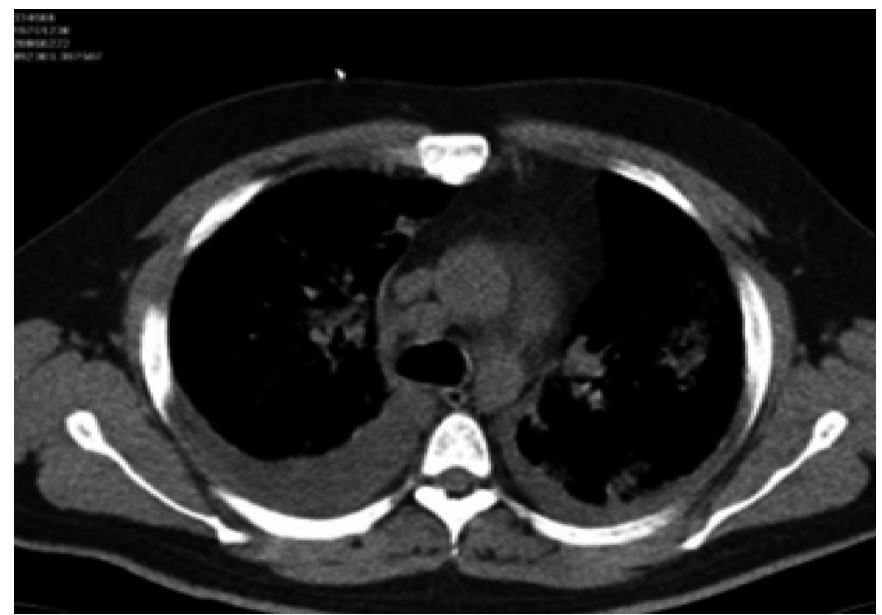

Fig. 1. Adenopatías de tamaño patológico pretraqueal, derrame pleural bilateral.

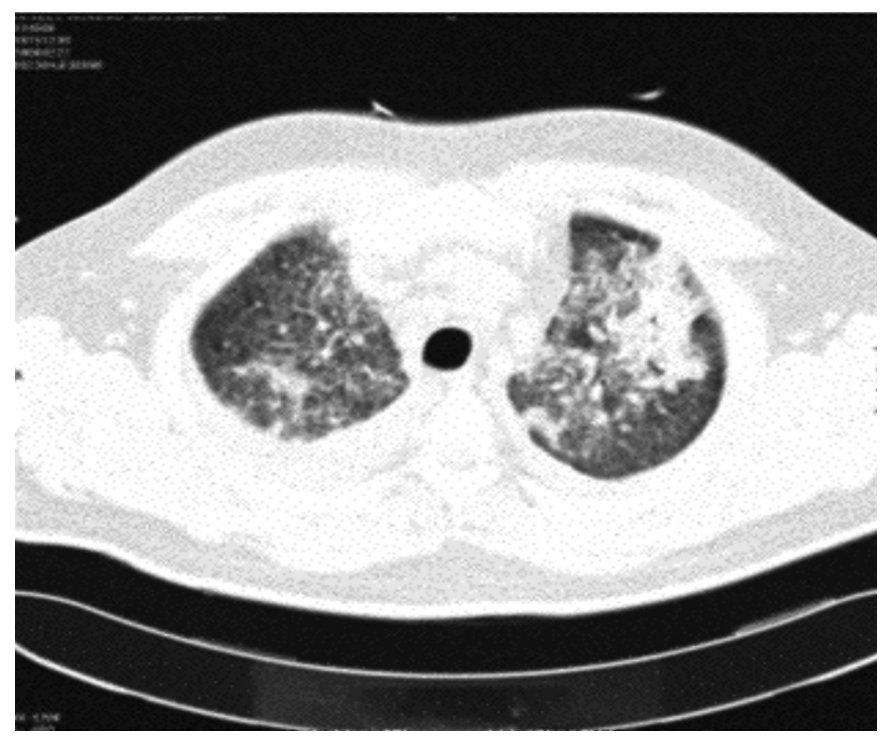

Fig. 2. Patrón intersticial retículo nodular septal y centro-lobulillar con áreas de condensación alveolar parcheada de distribución predominante en segmentos apicales.

de tórax no mostró cambios, por lo que se realizó un TAC torácico, que reveló el patrón en vidrio deslustrado y un derrame pleural bilateral moderado.

Se decidió realizar una biopsia pulmonar para obtener el diagnóstico. El informe fue de permeación linfática difusa (siguiendo un patrón intersticial nodular) por un adenocarcinoma acinar-papilar, bien diferenciado con abundantes cuerpos de psamoma. En el líquido pleural se observaban células con carácter citológicos de malignidad compatibles con adenocarcinoma. Se realizaron pruebas de histoquímica confirmando el origen pulmonar del adenocarcinoma.

Actualmente se encuentra en tratamiento con quimioterapia con la combinación cisplatino y gemcitabina.

Se han administrado hasta este momento dos ciclos, por lo que todavía no tenemos datos sobre la posible respuesta.

\section{DISCUSIÓN}

La incidencia del cáncer de pulmón se incrementa rápidamente a partir de los 50 años de edad. Algunos estudios han demostrado que los pacientes jóvenes con cáncer de pulmón 
presentan una enfermedad mucho más agresiva en comparación con los pacientes de mayor edad.

El cáncer de pulmón en adultos jóvenes es raro y posee algunas características que lo diferencian de las neoplasias malignas pulmonares es edades mayores, relativas a la distribución por sexos, tipo histológico y factores de riesgo. Las neoplasias malignas en menores de 45 años son poco frecuentes, alcanzando el 3\% de los casos de cáncer de pulmón (2).

El tipo histológico más frecuente en estos pacientes es el adenocarcinoma en el $54 \%$ de los casos en comparación con el $42 \%$ en los pacientes de más edad; seguido del carcinoma de células pequeñas con el $22 \%$ de los casos, frente al $15 \%$ en los enfermos de mayor edad, sin embargo el carcinoma escamoso se presentó únicamente en el $8 \%$ de los casos en los enfermos de menos de 40 años, frente al $36 \%$ en los de más de 55 años (3).

Las diferencias en la distribución por sexos existentes en los adultos son menores (4).

El principal factor de riesgo es el hábito tabáquico, existiendo una relación directamente proporcional al número de cigarrillos, duración del hábito y edad de inicio, sin diferencias entre sexos.

Algunos estudios clínicos y epidemiológicos han sugerido la existencia de un componente de agregación familiar en el cáncer de pulmón en pacientes jóvenes, Kreuzer et al realizarón un estudio de casos y controles, dividiendo a la población en dos grupos en función de la edad (menores de 45 años y entre 55 y 69 años). Dicho estudio mostró una frecuencia mayor de casos de cáncer pulmonar en familiares de primer grado en el grupo de menor edad con respecto al grupo control $\mathrm{y}$ al grupo de pacientes mayores (5).

La clínica que presentaban los pacientes jóvenes con cáncer de pulmón fue de tos, dolor torácico y disnea.

Según algunos autores, la presentación como enfermedad diseminada en el momento del diagnóstico es más frecuente entre los adultos jóvenes, así como el menor grado de diferenciación histológica (2). No existe consenso en la literatura con respecto a la supervivencia global en los pacientes menores de 40 años. Liu et al no encontraron diferencias en la superviven- cia global ni en el tiempo hasta la progresión en un análisis sobre 157 pacientes menores de 40 años en comparación con un grupo de mayores de 50 años con un adenocarcinoma de pulmón (6). Por este motivo se aconseja un mismo tratamiento y manejo independientemente de la edad. Algunos estudios sugieren un mejor pronóstico en el grupo de pacientes jóvenes, que se explica por la existencia de patología asociada en los pacientes mayores, lo que incrementaría de manera directa la mortalidad y en ocasiones dificultaría o incluso impediría la utilización de fármacos antitumorales, que por otra parte serían muy mal tolerados en ocasiones por estos pacientes de mayor edad (7).

Algunos autores han observado una mayor supervivencia en las mujeres con cáncer respecto de los varones; pero en la mayoría de los trabajos no se ha demostrado una significación estadística en éste parámetro (8).

El adenocarcinoma de pulmón es una entidad patológica bien definida. La Organización Mundial de la Salud lo clasifica en acinares, sólidos, papilares y bronquio-alveolares. En ocasiones el adenocarcinoma de pulmón se presenta con una estructura histológica en la que predominan la inflamación y la fibrosis, siendo entonces difícil diferenciarlo de una enfermedad pulmonar intersticial difusa (EPID) (9). La coexistencia de un adenocarcinoma de pulmón moderadamente diferenciado con diseminación linfática bilateral, y asociado a una intensa reacción fibrosa intersticial, es una apariencia histológica que no está recogida como variante histológica en la última clasificación de la Organización Mundial de la Salud, aunque está morfología ha sido previamente publicada (9). Esta forma de presentación del adenocarcinoma pulmonar plantea la disyuntiva de si el tumor crece sobre una enfermedad intersticial previa (10) o es una forma de presentación anormal de reacción del pulmón ante la diseminación neoplásica.

Este tipo de hallazgos son una razón más para insistir en la necesidad del diagnóstico histológico en las EPID, bien mediante la práctica de una biopsia transbronquial o, si fuera necesario de una biopsia pulmonar abierta (como ocurrió en el paciente que hemos presentado).

\section{Bibliografía}

1. Moguer Mediavilla M, Fernández Freire A, Sabino Álvarez A . Cáncer de pulmón no microcítico. Medicine 2004; 9: 1550-62.

2. Ramalingam S, Pawlish K, Gadgeel S, Demers R, Kalemkerian GP, et al. Lung cancer in young patients: analysis of a surveillance, epidemiology, and end results database. J Clin Oncol 1998; 16: 651-7.

3. Marcos Sánchez F, Albo Castaño I , Juárez Ucelay F, Celdrán Gil J, Granados Lucena D, Durán Pérez-Navarro A. Adenocarcinoma pulmonar de evolución rapidamente fatal en un paciente de 28 años de edad. Investig Clin 2000; 3: 242-45.

4. Liu NS, Spizt MR, Kemp BL, Cooksley C, Fossella FV, Lee TS, et al. Adenocarcinoma of the lung in young patients: the M.D. Anderson experience. Cancer 2000; 88: 1837-41.

5. Kreuzer M, Kreienbrock L, Gerken M, Heinrich J, Bruske-Hohlfeld I, Muller KM, et al. Risk factors for lung cancer in young adults. Am J Epidemiol 1998; 147: 1028-37.

6. Bourke W, Milstein D, Giura R, Donghi M, Luisetti M, Rubin AH, et al. Lung cancer in young adults. Chest 1992; 102: 1723-9.

7. Albain K, Crowley J, LeBlanc M, Livingston R. Survival determinants in extensive-stage non-small cell lung cancer: The Southwest oncology group experience. J Clin Oncol 1991; 9: 1618-26.

8. Espinosa E, González Barón M, Feliu J. Factores pronósticos de respuesta y de supervivencia en los carcinomas no microcíticos de pulmón. En Factores pronósticos en Oncología. Editorial Interamericana Mc Graw-Hill. Madrid 1994; 73-82.

9. Lantuejoul S, Colby TV, Ferretti GR, Brichon PY, Brambilla C, Brambilla E. Adenocarcinoma of the lung mimicking inflammatory lung disease with honeycombing. Eur Respir J 2004; 24: 502-5.

10. Bouros D, Hatzakis K, Labrakis H, Zeibecoglon K. Association of malignancy with diseases causing interstitial pulmonary changes. Chest 2002; 121: 1278-89. 